\title{
ON SLICEWISE MONOTONE PARAMETERIZED PROBLEMS AND OPTIMAL PROOF SYSTEMS FOR TAUT
}

\author{
YIJIA CHEN AND JÖRG FLUM
}

\begin{abstract}
For a reasonable sound and complete proof calculus for first-order logic consider the problem to decide, given a sentence $\varphi$ of first-order logic and a natural number $n$, whether $\varphi$ has no proof of length $\leq n$. We show that there is a nondeterministic algorithm accepting this problem which, for fixed $\varphi$, has running time bounded by a polynomial in $n$ if and only if there is an optimal proof system for the set TAUT of tautologies of propositional logic. This equivalence is an instance of a general result linking the complexity of so-called slicewise monotone parameterized problems with the existence of an optimal proof system for TAUT.
\end{abstract}

\section{Introduction}

In this paper we relate the existence of optimal proof systems for the class TAUT of tautologies of propositional logic with the complexity of slicewise monotone parameterized problems. A proof system in the sense of Cook and Reckhow [4], say for the class TAUT, is a polynomial time computable function defined on $\{0,1\}^{*}$ and with TAUT as range. A proof system $P$ is optimal if for any other proof system $P^{\prime}$ for TAUT there is a polynomial $p \in \mathbb{N}[X]$ such that for every tautology $\alpha$, if $\alpha$ has a proof of length $n$ in $P^{\prime}$, then $\alpha$ has a proof of length $\leq p(n)$ in $P{ }^{1}$ In their fundamental paper [9] Krajíček and Pudlák showed that an optimal proof system for TAUT exists if $\mathrm{NE}=$ co-NE and they derived a series of statements equivalent to the existence of such an optimal proof system; however they conjectured that there is no optimal proof system for TAUT.

On the other hand, Gödel in a letter to von Neumann of 1956 (see [6]) asked for the complexity of the problem to decide, given a sentence $\varphi$ of first-order logic and a natural number $n$, whether $\varphi$ has a proof of length $\leq n$. In our study [2] of this problem we introduced the parameterized problem

$p$-GÖDEL

Instance: A first-order sentence $\varphi$ and $n \in \mathbb{N}$ in unary.

Parameter: $|\varphi|$.

Problem: Does $\varphi$ have a proof of length $\leq n$ ?

\footnotetext{
${ }^{1}$ All notions will be defined in a precise manner in later sections.
} 
Here we refer to any reasonable sound and complete proof calculus for first-order logic. We do not allow proof calculi, which, for example, admit all first-order instances of propositional tautologies as axioms (as then it would be difficult to recognize correct proofs if $\mathrm{P} \neq \mathrm{NP}$ ).

In a different context, namely when trying to show that a certain logic $L_{\leq}$for PTIME (introduced in [7]) does not satisfy some effectivity condition, Nash et al. introduced implicitly [12] (and this was done explicitly in [1]) the parameterized acceptance problem - $-\mathrm{ACC}_{\leq}$for nondeterministic Turing machines:

$p-\mathrm{ACC}_{\leq}$

Instance: A nondeterministic Turing machine $\mathbb{M}$ and $n \in \mathbb{N}$ in unary.

Parameter: $\|\mathbb{M}\|$, the size of $\mathbb{M}$.

Problem: Does $\mathbb{M}$ accept the empty input tape in $\leq n$ steps?

Both problems, $p$-GÖDEL and $p$ - $\mathrm{ACC}_{\leq}$, are slicewise monotone, that is, their instances have the form $(x, n)$, where $x \in\{0,1\}^{*}$ and $n \in \mathbb{N}$ is given in unary, ${ }^{2}$ the parameter is $|x|$, and finally for all $x \in\{0,1\}^{*}$ and $n, n^{\prime} \in \mathbb{N}$ we have

if $(x, n)$ is a positive instance and $n<n^{\prime}$, then $\left(x, n^{\prime}\right)$ is a positive instance.

A slicewise monotone problem is in the complexity class $\mathrm{XNP}_{\text {uni }}$ if there is a nondeterministic algorithm that accepts it in time $n^{f(|x|)}$ for some function

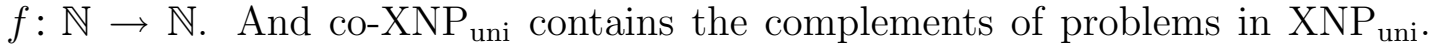
We show:

Theorem 1. TAUT has an optimal proof system if and only if every slicewise monotone problem in $\mathrm{NP}$ is in co- $\mathrm{XNP}_{\mathrm{uni}}$.

There are trivial slicewise monotone problems which are fixed-parameter tractable. However, for the slicewise monotone problems mentioned above we can show:

Theorem 2. TAUT has an optimal proof system $\Longleftrightarrow p-\mathrm{ACC}_{\leq} \in \mathrm{co}-\mathrm{XNP}_{\text {uni }}$ $\Longleftrightarrow p$-GÖDEL $\in$ CO-XNP uni

In [3] we showed that TAUT has a p-optimal proof system if and only if a certain $\operatorname{logic} L_{\leq}$is a P-bounded logic for P (=PTIME). The equivalence in the first line of Theorem 2 is the nondeterministic version of this result; in fact, an immediate consequence of it states that TAUT has an optimal proof system if and only if $L_{\leq}$is an NP-bounded logic for P (a concept that we will introduce in Section 6). It turns out that a slight variant of $L_{\leq}$is an NP-bounded logic for $\mathrm{P}$ (without any assumption).

\footnotetext{
${ }^{2}$ The requirement that $n$ is given in unary notation ensures that the classical complexity of most slicewise monotone problems we consider is in NP.
} 
The content of the different sections is the following. In Section 2 and Section 3 we recall the concepts and results of parameterized complexity and on optimal proof systems, respectively, we need in Section 4 to derive the equivalence in the first line of Theorem 2. Furthermore, in Section 3 we claim that every problem hard for EEXP under polynomial time reductions has no optimal proof system. In Section 5 we derive some basic properties of slicewise monotone problems, show that $p-\mathrm{ACC}_{\leq}$is of highest parameterized complexity among the slicewise monotone problems with classical complexity in NP, and finally show that all the slicewise monotone problems we consider in a certain sense have the same complexity (see Proposition 14 for the precise statement). This yields Theorem 1 and the remaining equivalence of Theorem 2. As already mentioned, in Section 6 we analyze the relationship of the existence of an optimal proof system for TAUT and the properties of the logic $L_{\leq}$.

\section{Some preliminaries}

In this section we recall some basic definitions and concepts from parameterized complexity and introduce the concept of slicewise monotone parameterized problem.

We denote the alphabet $\{0,1\}$ by $\Sigma$. The length of a string $x \in \Sigma^{*}$ is denoted by $|x|$. We identify problems with subsets $Q$ of $\Sigma^{*}$. Clearly, as done mostly, we present concrete problems in a verbal, hence uncodified form or by using other alphabets. We denote by $\mathrm{P}$ the class of problems $Q$ such that $x \in Q$ is solvable in time polynomial in $|x|$.

All deterministic and nondeterministic Turing machines have $\Sigma$ as their alphabet. If necessary we will not distinguish between a Turing machine and its code, a string in $\Sigma^{*}$. If $\mathbb{M}$ is a Turing machine we denote by $\|\mathbb{M}\|$ the length of its code.

Sometimes statements containing a formulation like "there is a $d \in \mathbb{N}$ such that for all $x \in \Sigma^{*}: \ldots \leq|x|^{d "}$ can be wrong for $x \in \Sigma^{*}$ with $|x| \leq 1$. We trust the reader's common sense to interpret such statements reasonably.

If $\mathbb{A}$ is any (deterministic or nondeterministic) algorithm and $\mathbb{A}$ accepts $x$, then we denote by $t_{\mathbb{A}}(x)$ the number of steps of a shortest accepting run of $\mathbb{A}$ on $x$; if $\mathbb{A}$ does not accept $x$, then $t_{\AA}(x)$ is not defined.

2.1. Parameterized complexity. We view parameterized problems as pairs $(Q, \kappa)$ consisting of a classical problem $Q \subseteq \Sigma^{*}$ and a parameterization $\kappa: \Sigma^{*} \rightarrow \mathbb{N}$, which is required to be polynomial time computable. We will present parameterized problems in the form we did it for $p$-GöDEL and $p-\mathrm{ACC}_{\leq}$in the Introduction.

A parameterized problem $(Q, \kappa)$ is fixed-parameter tractable (or, in FPT) if $x \in Q$ is solvable by an fpt-algorithm, that is, by a deterministic algorithm running in time $f(\kappa(x)) \cdot|x|^{O(1)}$ for some computable $f: \mathbb{N} \rightarrow \mathbb{N}$. 
Let $\mathrm{C}$ be a complexity class of classical complexity theory defined in terms of deterministic (nondeterministic) algorithms. A parameterized problem $(Q, \kappa)$ is in the class $\mathrm{XC}_{\text {uni }}$ if there is a deterministic (nondeterministic) algorithm deciding (accepting) $Q$ and witnessing for every $k \in \mathbb{N}$ that the classical problem

$$
(Q, \kappa)_{k}:=\{x \in Q \mid \kappa(x)=k\},
$$

the $k$ th slice of $(Q, \kappa)$, is in $\mathrm{C}$. For example, $(Q, \kappa)$ is in the class $\mathrm{XP}_{\text {uni }}$ if there is a deterministic algorithm $\mathbb{A}$ deciding $x \in Q$ in time $|x|^{f(\kappa(x))}$ for some function $f: \mathbb{N} \rightarrow \mathbb{N}$. And $(Q, \kappa)$ is in the class $\mathrm{XNP}_{\text {uni }}$ if there is a nondeterministic algorithm $\mathbb{A}$ accepting $Q$ such that for some function $f: \mathbb{N} \rightarrow \mathbb{N}$ we have $t_{\mathbb{A}}(x) \leq$ $|x|^{f(\kappa(x))}$ for all $x \in Q$. Finally, a parameterized problem $(Q, \kappa)$ is in the class co- $\mathrm{XC}_{\text {uni }}$ if its complement $\left(\Sigma^{*} \backslash Q, \kappa\right)$ is in $\mathrm{XC}_{\text {uni }}$.

We have added the subscript "uni" to the names of these classes to emphasize that they are classes of the so-called uniform parameterized complexity theory. If in the definition of $\mathrm{XP}_{\text {uni }}$ and $\mathrm{XNP}_{\text {uni }}$ we require the function $f$ to be computable, then we get the corresponding classes of the strongly uniform theory. For example, FPT is a class of this theory.

A parameterized problem $(Q, \kappa)$ is slicewise monotone if its instances have the form $(x, n)$, where $x \in \Sigma^{*}$ and $n \in \mathbb{N}$ is given in unary, if $\kappa((x, n))=|x|$, and finally if the slices are monotone, that is, for all $x \in \Sigma^{*}$ and $n, n^{\prime} \in \mathbb{N}$

$$
(x, n) \in Q \text { and } n<n^{\prime} \text { imply }\left(x, n^{\prime}\right) \in Q .
$$

We already remarked that the problems $p$-GöDEL and $p-\mathrm{ACC}_{\leq}$are slicewise monotone.

Clearly, every parameterized problem $(Q, \kappa)$ with $Q \in \mathrm{NP}$ is in $\mathrm{XNP}_{\text {uni }}$; thus we can replace co-XNP $\mathrm{Xni}_{\text {un }} \mathrm{XNP}_{\text {uni }} \cap$ co-XNP $\mathrm{Xni}_{\text {un }}$ everywhere in Theorem 1 and Theorem 2 .

\section{Optimal proof systems}

Let $Q \subseteq \Sigma^{*}$ be a problem. A proof system for $Q$ is a surjective function $P: \Sigma^{*} \rightarrow Q$ computable in polynomial time. Then, if $P(w)=x$, we say that $w$ is a $P$-proof of $x$. A proof system $P$ for $Q$ is optimal if for any other proof system $P^{\prime}$ for $Q$ there is a polynomial $p \in \mathbb{N}[X]$ such that for every $x \in Q$, if $x$ has a $P^{\prime}$-proof of length $n$, then $x$ has a $P$-proof of length $\leq p(n)$. Hence, any $P^{\prime}$-proof can be translated into a $P$-proof by a nondeterministic polynomial time algorithm.

The corresponding deterministic concept is the notion of p-optimality. The proof system $P$ for $Q$ is polynomially optimal or $p$-optimal if for every proof system $P^{\prime}$ for $Q$ there is a polynomial time computable $T: \Sigma^{*} \rightarrow \Sigma^{*}$ such that for all $w^{\prime} \in \Sigma^{*}$

$$
P\left(T\left(w^{\prime}\right)\right)=P^{\prime}\left(w^{\prime}\right)
$$

We list some known results. Part (1) and (2) are immediate from the definitions. 
(1) Every $p$-optimal proof system is optimal.

(2) Every nonempty $Q \in$ PTIME has a $p$-optimal proof system, every nonempty $Q \in \mathrm{NP}$ has an optimal proof system.

(3) ([8]) If $Q$ is nonempty and $Q \leq^{p} Q^{\prime}$ (that is, if $Q$ is polynomial time reducible to $Q^{\prime}$ ) and $Q^{\prime}$ has a (p-)optimal proof system, then $Q$ has a (p-)optimal proof system too.

(4) ([10]) Every $Q$ hard for $\operatorname{EXP}=\operatorname{DTIME}\left(2^{n^{O(1)}}\right)$ under polynomial time reductions has no p-optimal proof system.

It is not known whether there is a problem $Q \notin \mathrm{P}(Q \notin \mathrm{NP})$ with a p-optimal (an optimal) proof system. As mentioned in the Introduction, Krajícek and Pudlák [9] conjectured that there is no optimal proof system for the set TAUT of tautologies.

Concerning (4) we did not find a corresponding result for optimal proof systems in the literature. We can show:

Proposition 3. Every $Q$ hard for EEXP $=\operatorname{DTIME}\left(2^{2^{n^{O(1)}}}\right)$ under polynomial time reductions has no optimal proof system.

We do not need this result (and will prove it in the full version of the paper). However we state a consequence:

Corollary 4. There is no optimal proof system for the set of valid sentences of first-order logic.

3.1. Almost optimal algorithms and enumerations of P-easy subsets. Let $Q \subseteq \Sigma^{*}$ be a problem. A deterministic (nondeterministic) algorithm $\mathbb{A}$ accepting $Q$ is almost optimal or optimal on positive instances of $Q$ if for every deterministic (nondeterministic) algorithm $\mathbb{B}$ accepting $Q$ there is a polynomial $p \in \mathbb{N}[X]$ such that for all $x \in Q$

$$
t_{\mathbb{A}}(x) \leq p\left(t_{\mathbb{B}}(x)+|x|\right) .
$$

By definition a subset $Q^{\prime}$ of $Q$ is P-easy if $Q^{\prime} \in \mathrm{P}$. An enumeration of the $\mathrm{P}$-easy subsets of $Q$ by $\mathrm{P}$-machines (by NP-machines) is a computable function $M: \mathbb{N} \rightarrow \Sigma^{*}$ such that

(i) for every $i \in \mathbb{N}$ the string $M(i)$ is a deterministic (nondeterministic) Turing machine deciding (accepting) a P-easy subset of $Q$ in polynomial time;

(ii) for every P-easy subset $Q^{\prime}$ of $Q$ there is an $i \in \mathbb{N}$ such that $M(i)$ decides (accepts) $Q^{\prime}$.

If in the nondeterministic case instead of (i) we only require

$\left(\mathrm{i}^{\prime}\right)$ for every $i \in \mathbb{N}$ the string $M(i)$ is a nondeterministic Turing machine accepting a subset of $Q$ in polynomial time,

we obtain the notion of a weak enumeration of $\mathrm{P}$-easy subsets of $Q$ by NPmachines. 
We denote by TAUT the class of all tautologies of propositional logic. We need the following theorem:

Theorem 5. (1) The following statements are equivalent:

(a) TAUT has a p-optimal proof system.

(b) TAUT has an almost optimal deterministic algorithm.

(c) TAUT has an enumeration of the $\mathrm{P}$-easy subsets by $\mathrm{P}$-machines.

(2) The following statements are equivalent:

(a) TAUT has an optimal proof system.

(b) TAUT has an almost optimal nondeterministic algorithm.

(c) TAUT has a weak enumeration of the P-easy subsets by NP-machines.

(d) TAUT has an enumeration of the $\mathrm{P}$-easy subsets by NP-machines.

The equivalence of (a) and (b) in (1) and (2) is due to [9], the equivalence to (c) to [13]. The equivalence in (2) to (d) will be a by-product of the proof of Theorem 8; the equivalence was already claimed in [13] but its author was so kind to point out to us that he did not realize the difference between (c) and (d): some machines $M(i)$ of a weak enumeration might accept subsets of $Q$ which are not P-easy (but only in NP).

\section{Linking slicewise monotone problems and optimal proof systems}

The following result yields a uniform bound on the complexity of slicewise monotone problems whose complements have optimal proof systems.

Theorem 6. Let $(Q, \kappa)$ be a slicewise monotone parameterized problem with decidable $Q$.

(1) If $\Sigma^{*} \backslash Q$ has a p-optimal proof system, then $(Q, \kappa) \in \mathrm{XP}_{\text {uni }}$.

(2) If $\Sigma^{*} \backslash Q$ has an optimal proof system, then $(Q, \kappa) \in \mathrm{co}-\mathrm{XNP}_{\mathrm{uni}}$.

As by (3) on page 5 every nonempty problem in co-NP has a (p-)optimal proof system if TAUT has one, we immediately get:

Corollary 7. Let $(Q, \kappa)$ be a slicewise monotone parameterized problem with $Q$ in NP.

(1) If TAUT has a p-optimal proof system, then $(Q, \kappa) \in \mathrm{XP}_{\text {uni }}$.

(2) If TAUT has an optimal proof system, then $(Q, \kappa) \in \mathrm{co-XNP} \mathrm{Xni}_{\text {. }}$.

Concerning Theorem $6(1)$ we should mention that Monroe [11] has shown that if the complement of (the classical problem underlying) $p-\mathrm{ACC}_{\leq}$has an almost optimal algorithm (which by [9] holds if it has a p-optimal proof system), then $p-\mathrm{ACC}_{\leq} \in \mathrm{XP}_{\text {uni }}$.

Proof of Theorem 6: We present the proof for (2), the proof for (1) is obtained by the obvious modifications. Let $(Q, \kappa)$ be slicewise monotone and let $\mathbb{Q}$ be a deterministic algorithm deciding $Q$. Assume that $\Sigma^{*} \backslash Q$ has an optimal proof system. It is well-known [9] that then $\Sigma^{*} \backslash Q$ has an almost optimal nondeterministic algorithm $\mathbb{O}$. We have to show that $(Q, \kappa) \in$ co-XNP $\mathrm{Xni}_{\text {un }}$. 
Let $\mathbb{S}$ be the algorithm that, on $x \in \Sigma^{*}$, by systematically applying $\mathbb{Q}$ to the inputs $(x, 0),(x, 1), \ldots$ computes

$$
n(x):=\text { the least } n \text { such that }(x, n) \in Q .
$$

If $(x, n) \notin Q$ for all $n \in \mathbb{N}$, then $n(x)$ is not defined and $\mathbb{S}$ does not stop. We show that the following algorithm $\mathbb{A}$ witnesses that $\left(\Sigma^{*} \backslash Q, \kappa\right) \in \mathrm{XNP}_{\text {uni. }}$.

$$
\begin{array}{cc}
\mathbb{A}(x, n) & / / x \in \Sigma^{*}, n \in \mathbb{N} \text { in unary } \\
\text { 1. } & \text { In parallel simulate } \mathbb{S} \text { on input } x \text { and } \mathbb{O} \text { on input }(x, n) \\
\text { 2. } & \text { if } \mathbb{O} \text { accepts then accept } \\
\text { 3. } & \text { if } \mathbb{S} \text { stops, then } \\
\text { 4. } & \text { if } n<n(x) \text { then accept else reject. }
\end{array}
$$

By our assumptions on $\mathbb{O}$ and $\mathbb{S}$ and the slicewise monotonicity of $Q$, it should be clear that $\mathbb{A}$ accepts $\Sigma^{*} \backslash Q$. We have to show that $\mathbb{A}$ does it in the time required by $\mathrm{XNP}_{\text {uni. }}$. Hence, we have to determine the running time of $\mathbb{A}$ on inputs $(x, n) \notin Q$.

Case " $(x, \ell) \notin Q$ for all $\ell \in \mathbb{N}$ ": In this case $\mathbb{S}$ on input $x$ does not stop. Hence, the running time of $\mathbb{A}$ on input $(x, n)$ is determined by $\mathbb{O}$. The following algorithm $\mathbb{O}_{x}$ accepts $\Sigma^{*} \backslash Q$ : on input $(y, \ell)$ the algorithm $\mathbb{O}_{x}$ checks whether $y=x$. If so, it accepts and otherwise it runs $\mathbb{O}$ on input $(y, \ell)$ and answers accordingly. Clearly, for all $\ell \in \mathbb{N}$

$$
t_{\mathbb{O}_{x}}((x, \ell)) \leq O(|x|) .
$$

As $\mathbb{O}$ is almost optimal, we know that there is a constant $d_{x} \in \mathbb{N}$ (depending on $x)$ such that for all $(y, \ell) \in \Sigma^{*} \backslash Q$

$$
t_{\mathbb{O}}((y, \ell)) \leq\left(|(y, \ell)|+t_{\mathbb{O}_{x}}((y, \ell))\right)^{d_{x}} .
$$

In particular, we have

$$
t_{\mathbb{A}}((x, n))=O\left(t_{\mathbb{O}}((x, n))\right) \leq O\left((|(x, n)|+O(|x|))^{d_{x}}\right) \leq n^{d_{x}^{\prime}}
$$

for some constant $d_{x}^{\prime} \in \mathbb{N}$ (depending on $x$ ).

Case " $(x, \ell) \in Q$ for some $\ell \in \mathbb{N}$ ": Then $\mathbb{S}$ will stop on input $x$. Thus, in the worst case, $\mathbb{A}$ on input $(x, n)$ has to wait till the simulation of $\mathbb{S}$ on $x$ stops and then $\mathbb{A}$ must check whether the result $n(x)$ of the computation of $\mathbb{S}$ is bigger than $n$ or not and answer according to Line 4 . So in the worst case $\mathbb{A}$ takes time $O\left(t_{\mathbb{S}}(x)+O(n)\right) \leq n^{O\left(t_{\mathbb{S}}(x)\right)}$.

We show the equivalence in the first line of Theorem 2:

Theorem 8. (1) TAUT has a p-optimal proof system iff $p$ - $\mathrm{ACC}_{\leq} \in \mathrm{XP}_{\text {uni }}$.

(2) TAUT has an optimal proof system iff $p-\mathrm{ACC}_{\leq} \in \mathrm{Co}-\mathrm{XNP}_{\mathrm{uni}}$. 
Proof. Again we only prove (2) and by the previous corollary it suffices to show the corresponding implication from "right to left."

So assume that the complement of $p-\mathrm{ACC}_{<}$is in $\mathrm{XNP}_{\text {uni }}$ and let $\mathbb{A}$ be a nondeterministic algorithm witnessing it; in particular, $t_{\mathbb{A}}((\mathbb{M}, n)) \leq n^{f(\|\mathbb{M}\|)}$ for some function $f$ and all $(\mathbb{M}, n) \notin p-\mathrm{ACC}_{\leq}$. We show that TAUT has an enumeration of the P-easy subsets by NP-machines (and this suffices by Theorem 5).

We fix a deterministic Turing machine $\mathbb{M}_{0}$ that given a propositional formula $\alpha$ and an assignment checks if this assignment satisfies $\alpha$ in time $|\alpha|^{2}$.

For a deterministic Turing machine $\mathbb{M}$ let $\mathbb{M}^{*}$ be the nondeterministic machine that on empty input tape

- first guesses a propositional formula $\alpha$;

- then checks (by simulating $\mathbb{M}$ ) whether $\mathbb{M}$ accepts $\alpha$ and rejects if this is not the case;

- finally guesses an assignment and accepts if this assignment does not satisfy $\alpha$ (this is checked by simulating $\mathbb{M}_{0}$ ).

A deterministic Turing machine $\mathbb{M}$ is clocked if (the code of) $\mathbb{M}$ contains a natural number time $(\mathbb{M})$ such that $n^{\text {time(M) }}$ is a bound for the running time of $\mathbb{M}$ on inputs of length $n$ (in particular, a clocked machine is a polynomial time one).

Finally, for a clocked Turing machine $\mathbb{M}$ let $\mathbb{M}^{+}$be the nondeterministic Turing machine that on input $\alpha$ accepts if and only if (i) and (ii) hold:

(i) $\mathbb{M}$ accepts $\alpha$;

(ii) $\left(\mathbb{M}^{*},|\alpha|^{\text {time }(\mathbb{M})+4}\right) \notin p-\mathrm{ACC}_{\leq}$.

The machine $\mathbb{M}^{+}$checks (i) by simulating $\mathbb{M}$ and (ii) by simulating $\mathbb{A}$. Hence, if $\mathbb{M}^{+}$accepts $\alpha$, then

$$
t_{\mathbb{M}^{+}}(\alpha) \leq O\left(|\alpha|^{\text {time( }(\mathbb{M})}+t_{\mathbb{A}}\left(\left(\mathbb{M}^{*},|\alpha|^{\text {time }(\mathbb{M})+4}\right)\right)\right),
$$

and as $t_{\mathbb{A}}\left(\left(\mathbb{M}^{*},|\alpha|^{\text {time }(\mathbb{M})+4}\right)\right) \leq|\alpha|^{(\text {time }(\mathbb{M})+4) \cdot f\left(\left\|\mathbb{M}^{*}\right\|\right)}$, the Turing machine $\mathbb{M}^{+}$accepts in time polynomial in $|\alpha|$.

We show that $\mathbb{M}^{+}$, where $\mathbb{M}$ ranges over all clocked machines, yields an enumeration of all P-easy subsets of TAUT by NP-machines. First let $\mathbb{M}$ be a clocked machine. We prove that $\mathbb{M}^{+}$accepts a P-easy subset of TAUT.

$\mathbb{M}^{+}$accepts a subset of TAUT: If $\mathbb{M}^{+}$accepts $\alpha$, then, by (i), $\mathbb{M}$ accepts $\alpha$ and by (ii), $\left(\mathbb{M}^{*},|\alpha|^{\text {time }(\mathbb{M})+4}\right) \notin p$-ACC $\leq$. Therefore, by definition of $\mathbb{M}^{*}$, every assignment satisfies $\alpha$ and hence $\alpha \in$ TAUT.

$\mathbb{M}^{+}$accepts a P-easy set: If $\left(\mathbb{M}^{*}, m\right) \in p$ - $\mathrm{ACC}_{<}$for some $m$, then, by slicewise monotonicity of $p-\mathrm{ACC}_{\leq}$, the machine $\mathbb{M}^{+}$accepts a finite set and hence a P-easy set. If $\left(\mathbb{M}^{*}, m\right) \notin p$-ACC $\leq$ for all $m$, then $\mathbb{M}^{+}$accepts exactly those $\alpha$ accepted by $\mathbb{M}$; as $\mathbb{M}$ is clocked, this is a set in $\mathrm{P}$.

Now let $Q \subseteq$ TAUT be a P-easy subset of TAUT and let $\mathbb{M}$ be a clocked machine deciding $Q$. Then $\mathbb{M}^{+}$accepts $Q$. 


\section{Slicewise monotone parameterized problems}

In this section we observe that $p-\mathrm{ACC}_{<}$is a complete problem in the class of slicewise monotone parameterized problems with underlying classical problem in NP. Furthermore, we shall see that in Theorem 8 we can replace the problem $p-\mathrm{ACC}_{\leq}$ by other slicewise monotone parameterized problems (among them $p$-GöDEL) by showing for them that they are in the class $\mathrm{XP}_{\text {uni }}\left(\mathrm{co}-\mathrm{XNP}_{\text {uni }}\right)$ if and only if $p-\mathrm{ACC}_{\leq}$is.

5.1. The complexity of slicewise monotone problems. We start with some remarks on the complexity of slicewise monotone problems. In $[1,2]$ we have shown that $p-\mathrm{ACC}_{\leq}$and $p$-GÖDEL are not fixed-parameter tractable if "P $\neq$ NP holds for all time constructible and increasing functions," that is, if $\operatorname{DTIME}\left(h^{O(1)}\right) \neq \operatorname{NTIME}\left(h^{O(1)}\right)$ for all time constructible and increasing functions $h: \mathbb{N} \rightarrow \mathbb{N}$. However:

Proposition 9. (1) [2] Let $(Q, \kappa)$ be slicewise monotone. Then $(Q, \kappa)$ is nonuniformly fixed-parameter tractable, that is, there is a $c \in \mathbb{N}$, a function $f: \mathbb{N} \rightarrow \mathbb{N}$, and for every $k$ an algorithm deciding the slice $(Q, \kappa)_{k}$ in time $f(k) \cdot n^{c}$.

(2) Let $(Q, \kappa)$ be slicewise monotone with enumerable $Q$. Then $(Q, \kappa) \in \mathrm{XNP}_{\text {uni }}$.

Proof. (2) Let $\mathbb{Q}$ be an algorithm enumerating $Q$. The following algorithm shows that $(Q, \kappa) \in \mathrm{XNP}_{\text {uni }}$ : On input $(x, n)$ it guesses $m \in \mathbb{N}$ and a string $c$. If $c$ is the code of an initial segment of the run of $\mathbb{Q}$ enumerating $(x, m)$, then it accepts if $m \leq n$.

We remark that there are slicewise monotone problems with underlying classical problem of arbitrarily high complexity that are fixed-parameter tractable. In fact, let $Q_{0} \subseteq \Sigma^{*}$ be decidable. Then the slicewise monotone $(Q, \kappa)$ with

$$
Q:=\left\{(x, n) \mid x \in Q_{0}, n \in \mathbb{N} \text {, and }|x| \leq n\right\}
$$

(and $\kappa((x, n)):=|x|)$ is in FPT.

To compare the complexity of parameterized problems we use the standard notions of reduction that we recall first. Let $(Q, \kappa)$ and $\left(Q^{\prime}, \kappa^{\prime}\right)$ be parameterized problems. We write $(Q, \kappa) \leq^{\mathrm{fpt}}\left(Q^{\prime}, \kappa^{\prime}\right)$ if there is an fpt-reduction from $(Q, \kappa)$ to $\left(Q^{\prime}, \kappa^{\prime}\right)$, that is, a mapping $R: \Sigma^{*} \rightarrow \Sigma^{*}$ with:

(1) For all $x \in \Sigma^{*}$ we have $\left(x \in Q \Longleftrightarrow R(x) \in Q^{\prime}\right)$.

(2) $R(x)$ is computable in time $f(\kappa(x)) \cdot|x|^{O(1)}$ for some computable $f: \mathbb{N} \rightarrow \mathbb{N}$.

(3) There is a computable function $g: \mathbb{N} \rightarrow \mathbb{N}$ such that $\kappa^{\prime}(R(x)) \leq g(\kappa(x))$ for all $x \in \Sigma^{*}$.

We write $(Q, \kappa) \leq{ }^{\mathrm{xp}}\left(Q^{\prime}, \kappa^{\prime}\right)$ if there is an xp-reduction from $(Q, \kappa)$ to $\left(Q^{\prime}, \kappa^{\prime}\right)$, which is defined as $(Q, \kappa) \leq{ }^{\mathrm{fpt}}\left(Q^{\prime}, \kappa^{\prime}\right)$ except that instead of $(2)$ it is only required that $R(x)$ is computable in time $|x|^{f(\kappa(x))}$ for some computable $f: \mathbb{N} \rightarrow \mathbb{N}$. 
These are notions of reductions of the usual (strongly uniform) parameterized complexity theory. We get the corresponding notions $\leq_{\text {uni }}^{\mathrm{fpt}}$ and $\leq_{\mathrm{uni}}^{\mathrm{xp}}$ by allowing the functions $f$ and $g$ to be arbitrary (and not necessarily computable).

We shall use the following simple observation.

Lemma 10. If $(Q, \kappa) \leq \leq_{\text {uni }}^{\mathrm{xp}}\left(Q^{\prime}, \kappa^{\prime}\right)$ and $\left(Q^{\prime}, \kappa^{\prime}\right) \in \mathrm{XP}_{\text {uni }}$, then $(Q, \kappa) \in \mathrm{XP}_{\text {uni }}$. The same holds for $\mathrm{XNP}_{\text {uni }}$ instead of $\mathrm{XP}_{\mathrm{uni}}$.

We turn again to slicewise monotone problems. Among these problems with underlying classical problem in NP the problem $p-\mathrm{ACC}_{\leq}$is of highest complexity.

Proposition 11. Let $(Q, \kappa)$ be slicewise monotone and $Q \in \mathrm{NP}$. Then

$$
(Q, \kappa) \leq{ }^{\mathrm{fpt}} p-\mathrm{ACC}_{\leq} .
$$

Note that this result together with Theorem 8(2) yields Theorem 1.

Proof of Proposition 11: Let $\mathbb{M}$ be a nondeterministic Turing machine accepting $Q$. We may assume that for some $d \in \mathbb{N}$ the machine $\mathbb{M}$ on input $(x, n)$ performs exactly $|(x, n)|^{d}$ steps. For $x \in \Sigma^{*}$ let $\mathbb{M}_{x}$ be the nondeterministic Turing machine that on empty input tape, first writes $x$ on the tape, then guesses a natural number $m$, and finally simulates the computation of $\mathbb{M}$ on input $(x, m)$. We can assume that there is a polynomial time computable function $h$ such that $\mathbb{M}_{x}$ makes exactly $h(x, m) \in O\left(|x|+m+|(x, m)|^{d}\right)$ steps if it chooses the natural number $m$. Furthermore we can assume that $h(x, m)<h\left(x, m^{\prime}\right)$ for $m<m^{\prime}$.

Then $(x, n) \mapsto\left(\mathbb{M}_{x}, h(x, n)\right)$ is an fpt-reduction from $(Q, \kappa)$ to $p$ - $\mathrm{ACC}_{\leq}$: Clearly, if $(x, n) \in Q$ then $\left(\mathbb{M}_{x}, h(x, n)\right) \in p-\mathrm{ACC}_{\leq}$by construction of $\mathbb{M}_{x}$. Conversely, if $\left(\mathbb{M}_{x}, h(x, n)\right) \in p-\mathrm{ACC}_{\leq}$, then by the properties of $h$ we see that $\mathbb{M}$ accepts $(x, m)$ for some $m \leq n$. Thus, $(x, m) \in Q$ and therefore $(x, n) \in Q$ by slicewise monotonicity.

Later on we shall use the following related result.

Proposition 12. Let $(Q, \kappa)$ be slicewise monotone and assume that there is a nondeterministic algorithm $\mathbb{A}$ accepting $Q$ such that $t_{\mathbb{A}}(x, n) \leq n^{f(|x|)}$ for some time constructible $f$ and all $(x, n) \in Q$. Then

$$
(Q, \kappa) \leq{ }^{\mathrm{xp}} p-\mathrm{ACC}_{\leq}
$$

Proof. Let $\left(Q^{\prime}, \kappa^{\prime}\right)$ be the problem

Instance: $\quad x \in \Sigma^{*}$ and $m \in \mathbb{N}$ in unary.

Parameter: $|x|$.

Problem: Is there an $n \in \mathbb{N}$ such that $n^{f(|x|)} \leq m$ and $(x, n) \in Q$ ?

By the previous proposition we get our claim once we have shown:

(1) $\left(Q^{\prime}, \kappa^{\prime}\right)$ is slicewise monotone and $Q^{\prime} \in \mathrm{NP}$.

(2) $(Q, \kappa) \leq^{\operatorname{xp}}\left(Q^{\prime}, \kappa^{\prime}\right)$ 
To see (1) let $\mathbb{A}$ be as stated above and let $\mathbb{T}$ an algorithm witnessing the time constructibility of $f$; that is, $\mathbb{T}$ on input $k \in \mathbb{N}$ computes $f(k)$ in exactly $f(k)$ steps. An algorithm $\mathbb{B}$ witnessing that $Q^{\prime} \in \mathrm{NP}$ runs as follows on input $(x, m)$ :

- $\mathbb{B}$ guesses $n \in \mathbb{N}$;

- if $n=1$, the algorithm $\mathbb{B}$ rejects in case $m=0$;

if $n \geq 2$, the algorithm $\mathbb{B}$ simulates $m$ steps of the computation of $\mathbb{T}$ on input $|x|$; if thereby $\mathbb{T}$ does not stop, $\mathbb{B}$ rejects; otherwise, the simulation yields $f(|x|)$ and $\mathbb{B}$ checks whether $n^{f(|x|)}>m$ (this can be detected in time $O(m))$; in the positive case $\mathbb{B}$ rejects;

- finally $\mathbb{B}$ simulates the computation of $\mathbb{A}$ on $(x, n)$ and answers accordingly.

(2) Note that the mapping $(x, n) \mapsto\left(x, n^{f(|x|)}\right)$ is an xp-reduction.

5.2. Slicewise monotone problems related to logic. In the next section we will use some further slicewise monotone problems related to first-order logic and least fixed-point logic that we introduce now.

We assume familiarity with first-order logic FO and its extension least fixed-point logic LFP (e.g, see [5]). We denote by FO $\tau]$ and LFP $[\tau]$ the set of sentences of vocabulary $\tau$ of $\mathrm{FO}$ and of LFP, respectively. In this paper all vocabularies are finite sets of relational symbols.

If the structure $\mathcal{A}$ is a model of the LFP-sentence $\varphi$ we write $\mathcal{A} \models \varphi$. We only consider structures $\mathcal{A}$ with finite universe $A$. The size $\|\mathcal{A}\|$ of the structure $\mathcal{A}$ is the length of a reasonable encoding of $\mathcal{A}$ as string in $\Sigma^{*}$. An algorithm based on the inductive definition of the satisfaction relation for LFP shows (see [14]):

Proposition 13. The model-checking problem $\mathcal{A} \models \varphi$ for structures $\mathcal{A}$ and LFPsentences $\varphi$ can be solved in time $\|\mathcal{A}\|^{O(|\varphi|)}$.

Let $L=\mathrm{FO}$ or $L=\mathrm{LFP}$. First we introduce the parameterized problem

$p$ - $L$-MODEL

Instance: An $L$-sentence $\varphi$ and $n \in \mathbb{N}$ in unary.

Parameter: $|\varphi|$.

Problem: Is there a structure $\mathcal{A}$ with $\mathcal{A} \vDash \varphi$ and

$|A| \leq n$ ?

Here, $|A|$ denotes the size of the universe $A$ of $\mathcal{A}$. For every vocabulary $\tau$ we let $\tau_{<}:=\tau \cup\{<\}$, where $<$ is a binary relation symbol not in $\tau$. For $m \geq 1$ we say that an $L\left[\tau_{<}\right]$-sentence $\varphi$ is $\leq m$-invariant if for all $\tau$-structures $\mathcal{A}$ with $|A| \leq m$ we have

$$
\left(\mathcal{A},<_{1}\right) \vDash \varphi \Longleftrightarrow\left(\mathcal{A},<_{2}\right) \vDash \varphi
$$

for all orderings $<_{1}$ and $<_{2}$ on $A$.

Finally we introduce the slicewise monotone parameterized problem 


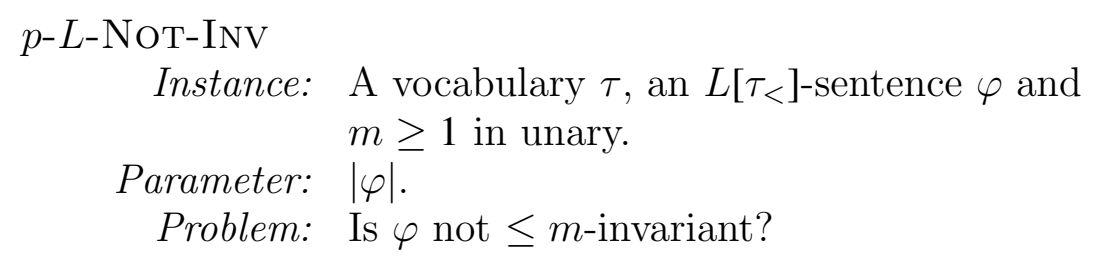

5.3. Membership in $\mathrm{XP}_{\text {uni }}$ and co- $\mathrm{XNP}_{\text {uni }}$. Concerning membership in the classes $\mathrm{XP}_{\text {uni }}$ and co- $\mathrm{XNP}_{\text {uni }}$ all the slicewise monotone problems we have introduced behave in the same way:

Proposition 14. Consider the parameterized problems

$$
\begin{aligned}
& p \text {-GÖDEL, } p \text {-FO-MODEL, } p \text {-LFP-MODEL, } p \text {-FO-NoT-INV, } \\
& p \text {-LFP-NOT-INV, and } p \text {-ACC } \leq \text {. }
\end{aligned}
$$

If one of the problems is in $\mathrm{XP}_{\mathrm{uni}}$, then all are; if one of the problems is in co- $\mathrm{XNP}_{\mathrm{uni}}$, then all are.

By Theorem 8 this result yields Theorem 2. We prove it with Lemmas 15-18.

Lemma 15. ([2]) $p$-GÖDEL $\leq{ }^{\mathrm{fpt}} p$-FO-MODEL.

Lemma 16. Let $L=\mathrm{FO}$ or $L=\mathrm{LFP}$. Then $p-L$-MODEL $\leq^{\mathrm{fpt}} p-L-\mathrm{NOT}-\mathrm{INV}$.

Proof. Let $\varphi$ be a sentence of vocabulary $\tau$ We set $\tau^{\prime}:=\tau \cup\{P\}$ with a new unary relation symbol $P$ and consider the sentence of vocabulary $\tau_{<}^{\prime}$

$$
\psi(\varphi):=\varphi \wedge \text { "P holds for the first element of }<\text {." }
$$

Clearly, for every $n \geq 2$

$$
(\varphi, n) \in p \text {-FO-MODEL } \Longleftrightarrow(\psi(\varphi), n) \in p \text {-FO-NOT-INV }
$$

and the same equivalence holds for $p$-LFP-ModeL and $p$-LFP-Not-Inv. Thus $(\varphi, n) \mapsto(\psi(\varphi), n)$ is the desired reduction in both cases.

Lemma 17. $p$-LFP-NoT-INV $\leq^{\mathrm{xp}} p-\mathrm{ACC}_{\leq}$

Proof. Consider the algorithm $\mathbb{A}$ that on input $(\varphi, m)$, where $\varphi$ is an LFP-sentence and $m \geq 1$, guesses a structure $\mathcal{A}$ and two orderings $<_{1}$ and $<_{2}$ and accepts if $|A| \leq m,\left(\mathcal{A},<_{1}\right) \vDash \varphi$, and $\left(\mathcal{A},<_{2}\right) \vDash \neg \varphi$. Then, by Proposition 13 , the algorithm $\mathbb{A}$ witnesses that $p$-LFP-NoT-INV satisfies the assumptions on $(Q, \kappa)$ in Proposition 12. This yields the claim.

Lemma 18. (1) If $p$-GÖDEL $\in \mathrm{XP}_{\text {uni }}$, then $p-\mathrm{ACC}_{\leq} \in \mathrm{XP}_{\text {uni }}$.

(2) If $p$-GöDEL $\in$ Co- $\mathrm{XNP}_{\text {uni }}$, then $p-\mathrm{ACC}_{\leq} \in \mathrm{co}-\mathrm{XNP}_{\text {uni }}$.

Proof. We give the proof of (2). By standard means we showed in [2, Lemma 7$]$ that there exists a $d \in \mathbb{N}$ and a polynomial time algorithm that assigns to every nondeterministic Turing machine $\mathbb{M}$ a first-order sentence $\varphi_{\mathbb{M}}$ such that for $n \in \mathbb{N}$

$$
(\mathbb{M}, n) \in p-\mathrm{ACC}_{\leq} \Longrightarrow\left(\varphi_{\mathbb{M}}, n^{d}\right) \in p-\mathrm{GÖDEL} .
$$


Moreover,

$\varphi_{\mathbb{M}}$ has a proof $\Longrightarrow \mathbb{M}$ accepts the empty input tape.

Now assume that $\mathbb{A}$ is an algorithm that witnesses that the complement of $p$-GöDEL is in $\mathrm{XNP}_{\text {uni. }}$. We may assume that every run of $\mathbb{A}$ either accepts its input or is infinitely long. Let $d \in \mathbb{N}$ be as above. We present an algorithm $\mathbb{B}$ showing that the complement of $p-\mathrm{ACC}_{<}$is in $\mathrm{XNP}_{\text {uni }}$. On input $(\mathbb{M}, n)$ the algorithm $\mathbb{B}$ first computes $\varphi_{\mathbb{M}}$ and then runs two algorithms in parallel:

- a brute force algorithm that on input $\mathbb{M}$ searches for the least $n_{\mathbb{M}}$ such that $\mathbb{M}$ on empty input tape has an accepting run of length $n_{\mathbb{M}}$;

- the algorithm $\mathbb{A}$ on input $\left(\varphi_{\mathbb{M}}, n^{d}\right)$.

If the brute force algorithm halts first and outputs $n_{\mathbb{M}}$, then $\mathbb{B}$ checks whether $n_{\mathbb{M}} \leq n$ and answers accordingly.

Assume now that $\mathbb{A}$ halts first. Then $\mathbb{A}$ accepts $\left(\varphi_{\mathbb{M}}, n^{d}\right)$ and $\left(\left(\varphi_{\mathbb{M}}, n^{d}\right) \notin\right.$ $p$-GöDEL and hence $(\mathbb{M}, n) \notin p$ - $\mathrm{ACC}_{\leq}$by $(1)$ and therefore) $\mathbb{B}$ accepts.

The algorithm $\mathbb{B}$ accepts the complement of $p-\mathrm{ACC}_{\leq}$; note that if no run of $\mathbb{A}$ accepts $\left(\varphi_{\mathbb{M}}, n^{d}\right)$, then $\left(\varphi_{\mathbb{M}}, n^{d}\right) \in p$-GöDEL and therefore $\mathbb{M}$ accepts the empty input tape by (2), so that in this case the computation of the brute force algorithm eventually will stop.

It remains to see that $\mathbb{B}$ accepts the complement of $p-\mathrm{ACC}_{<}$in the time required by $\mathrm{XNP}_{\text {uni }}$. We consider two cases.

$\mathbb{M}$ halts on empty input tape: Then an upper bound for the running time is given by the time that the brute force algorithm needs to compute $n_{\mathbb{M}}$ (and the time for the check whether $n_{\mathbb{M}} \leq n$ ); hence we have an upper bound of the form $n^{c_{\mathbb{M}}}$.

$\mathbb{M}$ does not halt on empty input tape: Then, by $(2)$, we have $\left(\varphi_{\mathbb{M}}, n^{d}\right) \notin p$-GöDEL; hence an upper bound is given by the running time of $\mathbb{A}$ on input $\left(\varphi_{\mathbb{M}}, n^{d}\right)$.

It should be clear that Lemmas 15-18 together with Lemma 10 yield a proof of Proposition 14.

\section{Optimal algorithms and the logic $L_{\leq}$}

In this section we interpret Theorem 2 in terms of the expressive power of a certain logic.

For our purposes a logic $L$ consists

- for every vocabulary $\tau$ of a set $L[\tau]$ of strings, the set of $L$-sentences of vocabulary $\tau$ and of an algorithm that for every vocabulary $\tau$ and every string $\xi$ decides whether $\xi \in L[\tau]$ (in particular, $L[\tau]$ is decidable for every $\tau$ );

- of a satisfaction relation $\models_{L}$; if $(\mathcal{A}, \varphi) \in \models_{L}$, written $\mathcal{A} \models_{L} \varphi$, then $\mathcal{A}$ is a $\tau$-structure and $\varphi \in L[\tau]$ for some vocabulary $\tau$; furthermore for each 
$\varphi \in L[\tau]$ the class $\operatorname{Mod}_{L}(\varphi):=\left\{\mathcal{A} \mid \mathcal{A} \vDash_{L} \varphi\right\}$ of models of $\varphi$ is closed under isomorphisms.

Definition 19. Let $L$ be a logic.

(a) $L$ is a logic for $\mathrm{P}$ if for all vocabularies $\tau$ and all classes $C$ (of encodings) of $\tau$-structures closed under isomorphisms we have

$$
C \in \mathrm{P} \quad \Longleftrightarrow C=\operatorname{Mod}_{L}(\varphi) \text { for some } \varphi \in L[\tau] .
$$

(b) $L$ is a $\mathrm{P}$-bounded logic for $\mathrm{P}$ if (a) holds and if there is an algorithm $\mathbb{A}$ deciding $\models_{L}$ (that is, for every structure $\mathcal{A}$ and $L$-sentence $\varphi$ the algorithm $\mathbb{A}$ decides whether $\mathcal{A} \models_{L} \varphi$ ) and if moreover, for fixed $\varphi$ the algorithm $\mathbb{A}$ runs in time polynomial in $\|\mathcal{A}\|$.

The relationship of these concepts with topics of this paper is already exemplified by the following simple observation.

Proposition 20. Let $L$ be a logic for $\mathrm{P}$ and define $p-\models_{L}$ by

$$
\begin{aligned}
p-\models_{L} \quad & \\
\text { Instance: } & \text { A structure } \mathcal{A} \text { and an } L \text {-sentence } \\
& \varphi . \\
\text { Parameter: } & |\varphi| . \\
\text { Problem: } & \text { Is } \mathcal{A} \models_{L} \varphi
\end{aligned}
$$

Then $L$ is a $\mathrm{P}$-bounded logic for $\mathrm{P}$ if and only if $p-\models_{L} \in \mathrm{XP}_{\mathrm{uni}}$.

This relationship suggests the following definition.

Definition 21. $L$ is an NP-bounded logic for $\mathrm{P}$ if it is a logic for $\mathrm{P}$ and $p$ $\models_{L} \in \mathrm{XNP}_{\text {uni }}$.

We introduce the logic $L_{\leq}$, a variant of LFP. ${ }^{3}$ For every vocabulary $\tau$ we set

$$
L_{\leq}[\tau]=\operatorname{LFP}\left[\tau_{<}\right]
$$

(recall that $\tau_{<}:=\tau \cup\{<\}$, with a new binary $<$ ) and define the semantics by

$$
\mathcal{A} \models_{L_{\leq}} \varphi \Longleftrightarrow(\varphi \text { is } \leq|A| \text {-invariant and }
$$

$$
\left.(\mathcal{A},<) \models_{\text {LFP }} \varphi \text { for some ordering }<\text { on } A\right) .
$$

Hence, by the previous proposition and the definition of $\models_{L_{\leq}}$, we get:

Proposition 22. (1) The following statements are equivalent:

- $L_{\leq}$is a $\mathrm{P}$-bounded logic for $\mathrm{P}$.

$-p-\models_{L_{\leq}} \in \mathrm{XP}_{\text {uni }}$.

$-p$-LFP-Not-Inv $\in \mathrm{XP}_{\text {uni }}$.

\footnotetext{
${ }^{3}$ In this section, if the structure $\mathcal{B}$ is a model of an LFP-sentence $\varphi$ we write $\mathcal{A} \models_{\text {LFP }} \varphi$ instead of $\mathcal{A}=\varphi$.
} 
(2) The following statements are equivalent:

- $L_{\leq}$is an NP-bounded logic for $\mathrm{P}$.

$-p-\models_{L_{\leq}} \in \mathrm{XNP}_{\text {uni }}$.

$-p$-LFP-Not-INV $\in$ co-XNP $\mathrm{Xni}_{\text {. }}$.

By Theorem 2 and Proposition 14 we get:

Theorem 23. TAUT has an optimal proof system if and only if $L_{\leq}$is an NPbounded logic for $\mathrm{P}$.

Hence, if TAUT has an optimal proof system, then there is an NP-enumeration of P-easy classes of graphs closed under isomorphisms. We do not define the concept of NP-enumeration explicitly, however the enumeration obtained by applying the algorithm in $\mathrm{XNP}_{\text {uni }}$ for $p$ - $\vDash_{L_{<}}$to the classes $\operatorname{Mod}_{L_{\leq}}(\varphi(\mathrm{GRAPH}) \wedge \psi)$, where $\varphi$ (GRAPH) axiomatizes the class of graphs and $\psi$ ranges over all sentences of $L_{\leq}$ in the language of graphs, is such an NP-enumeration. Note that even without the assumption that TAUT has an optimal proof system we know that there is such an NP-enumeration of P-easy classes of graphs closed under isomorphisms, as the following variant $L_{\leq}$(not) of $L_{\leq}$is an NP-bounded logic for P. The logic $L_{\leq}$(not) has the same syntax as $L_{\leq}$and the semantics is given by the following clause:

$$
\mathcal{A} \models_{L_{\leq}(\mathrm{not})} \varphi \Longleftrightarrow \quad \operatorname{not} \mathcal{A} \models_{L_{\leq}} \varphi .
$$

As the class $\mathrm{P}$ is closed under complements, $L_{\leq}$(not) is a logic for P. And $L_{\leq}$(not) is an NP-bounded logic for $\mathrm{P}$, as $p$-LFP-Not-INV $\in \mathrm{XNP}_{\text {uni }}$.

Acknowledgement. This research has been partially supported by the National Nature Science Foundation of China (60970011), the Sino-German Center for Research Promotion (GZ400), and the John Templeton Foundation through Project \# 13152, the Infinity Project at the Centre de Recerca Matemàtica.

\section{References}

[1] Y. Chen and J. Flum. A logic for PTIME and a parameterized halting problem. In Proceedings of the 24th IEEE Symposium on Logic in Computer Science (LICS'O9), pages 397-406, 2009.

[2] Y. Chen and J. Flum. On the complexity of Gödel's proof predicate. The Journal of Symbolic Logic, 75(1): 239-254, 2010.

[3] Y. Chen and J. Flum. On p-optimal proof systems and logics for PTIME. In Proceedings of the 37th International Colloquium on Automata, Languages and Programming (ICALP'10, Track B), Lecture Notes in Computer Science 6199, pages 321-332, 2010.

[4] S. Cook and R. Reckhow. The relative efficiency of propositional proof systems. The Journal of Symbolic Logic, 44:36-50, 1979.

[5] H.-D. Ebbinghaus and J. Flum. Finite Model Theory, 2nd edition, Springer, 1999.

[6] K. Gödel. Collected Works, vol. VI, 372-376, Clarendon Press, 2003.

[7] Y. Gurevich. Logic and the challenge of computer science. In Current Trends in Theoretical Computer Science, Computer Science Press, 1-57, 1988. 
[8] J. Köbler and J. Messner. Complete problems for promise classes by optimal proof systems for test sets. In Proceedings of the 13th IEEE Conference on Computational Complexity (CCC' 98), 132-140, 1998.

[9] J. Krajíček and P. Pudlák. Propositional proof systems, the consistency of first order theories and the complexity of computations. The Journal of Symbolic Logic, 54:1063$1088,1989$.

[10] J. Messner. On optimal algorithms and optimal proof systems. In Proceedings of the 16th Symposium on Theoretical Aspects of Computer Science (STACS'99), Lecture Notes in Computer Science 1563:361-372, 1999.

[11] H. Monroe. Speedup for natural problems. Electronic Colloquium on Computational Complexity, Report TR09-056, 2009.

[12] A. Nash, J. Remmel, and V. Vianu. PTIME queries revisited. In Proceedings of the 10th International Conference on Database Theory (ICDT'05), T. Eiter and L. Libkin (eds.), Lecture Notes in Computer Science 3363:274-288, 2005.

[13] Z. Sadowski. On an optimal propositional proof system and the structure of easy subsets. Theoretical Computer Science, 288(1):181-193, 2002.

[14] M.Y. Vardi. On the complexity of bounded-variable queries. In Proceedings of the 14th ACM Symposium on Principles of Database Systems (PODS'95), pages 266-276, 1995.

YiJia Chen

Department of Computer Science

Shanghai Jiaotong University

CHINA

E-mail address: yijia.chen@cs.sjtu.edu.cn

JÖRG FLUM

Mathematisches Institut

Albert-Ludwigs Universität Freiburg

Germany

E-mail address: joerg.flum@math.uni-freiburg.de 